\title{
Die Revision der \\ Schweizer Pharmakodizes
}

\author{
Jürg Granwehr \\ lic. iur. RA, Bereichsleiter Pharma \& Recht, scienceindustries Zürich
}

Seit vielen Jahren besteht in der Schweiz eine über das Gesetz hinausgehende, international abgestimmte Pharma-Selbstregulierung, auf welche sich Firmen freiwillig verpflichten können. Trägerorganisation ist scienceindustries, deren Kodex-Sekretariat mit dem Vollzug der Kodizes betraut ist. Im Nachgang zur Revision des Heilmittelgesetzes, der Inkraftsetzung der Verordnung über die Integrität und Transparenz im Heilmittelbereich sowie der Code Consolidation des europäischen Pharmaverbandes wurden die Schweizer Pharmakodizes umfassend überarbeitet. Die revidierten Kodizes werden am 1. Januar 2021 in Kraft treten. Nachfolgend werden ausgewählte Themen beleuchtet, die für die Ärzteschaft von speziellem Interesse sein könnten.

Während der Pharmakodex (PK) primär die Integritätsvorschriften regelt, enthält der Pharma-Kooperations-Kodex (PKK) die Vorgaben zur Offenlegung der geldwerten Zuwendungen an Fachpersonen, Gesundheitsversorgungs-Organisationen und Patientenorganisationen. In räumlicher Hinsicht finden beide Kodizes auf alle in ihnen geregelten Sachverhalte Anwendung, sofern sich diese in der Schweiz zutragen. Beim persönlichen Anwendungsbereich findet der PK

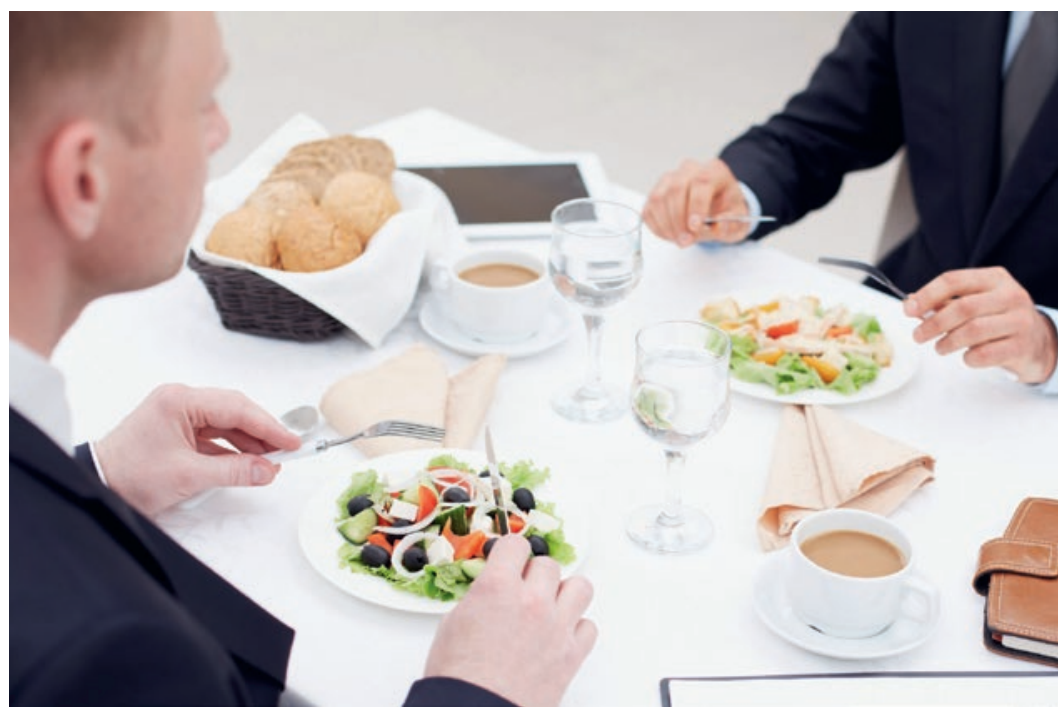

Zuwendungen für Mahlzeiten sind nur im Rahmen von Fachgesprächen oder Veranstaltungen zulässig und dürfen maximal 100 Franken kosten. mit Blick auf die Fachpersonen breite Anwendung (insbesondere sind auch Interaktionen mit Drogistinnen und Drogisten einbezogen). Der PKK ist indes auf Interaktionen im Kontext verschreibungspflichtiger Arzneimittel beschränkt, was den Kreis der Fachpersonen hier enger fasst. Denn die Offenlegung bezweckt, Transparenz mit Bezug auf allfällige Beeinflussungen des Verschreibungsverhaltens zu schaffen, weshalb Kooperationen im Kontext von verschreibungsfreien Arzneimitteln nicht unter den Anwendungsbereich des PKK fallen.

Beiden Kodizes ist gemeinsam, dass sie zu Beginn eine Reihe von Begriffen klären und dann dieselben Verhaltens- und Integritätsgrundsätze festschreiben.

Der PK regelt weiter die Fachwerbung und Information über Arzneimittel, wobei er oft detaillierter ist als die Arzneimittelwerbeverordnung. Die Durchführung von Veranstaltungen zur Arzneimittel-Fachwerbung nimmt einen prominenten Platz ein, wie auch die Unterstützung von medizinischer Fort- und Weiterbildung. In der revidierten Fassung des PK werden alsdann die Beratungs- und Dienstleistungsverträge mit Fachpersonen und Gesundheitsversorgungs-Organisation in einem hohen Detaillierungsgrad geregelt, wie auch die Zusammenarbeit und Unterstützung von $\mathrm{Pa}$ tientenorganisationen. Schliesslich enthält der PK eine Reihe von Vorschriften mit Bezug auf das Sponsoring klinischer Versuche. 
Der PKK regelt primär die Offenlegung der Kooperationszuwendungen im Zusammenhang mit verschreibungspflichtigen Arzneimitteln, die Ausnahmen von dieser Pflicht sowie die formalen Aspekte der Offenlegung.

\section{Vergleich zum gesetzlichen Rahmen}

Die Themen Integrität und Transparenz wurden bei der Revision des Heilmittelgesetzes (HMG) aus den Werbebestimmungen ausgegliedert und ein eigener Abschnitt dazu geschaffen (Art. 55 und 56 HMG). Dar-

\section{Das Bundesamt für Gesundheit ist neu für den Vollzug der Integritäts- und Transparenz- vorschriften zuständig.}

auf gestützt wurde dann die neue Verordnung über Integrität und Transparenz im Heilmittelbereich (VITH) erlassen. Gleichzeitig wurden die Zuständigkeiten neu geregelt: Der Vollzug der Werbebestimmungen ist nach wie vor bei Swissmedic angesiedelt; das Bundesamt für Gesundheit (BAG) ist neu für den Vollzug der Integritäts- und Transparenzvorschriften zuständig. Mit Blick auf den erläuternden Bericht des BAG zu Art. 3 VITH ist davon auszugehen, dass das selbstregulative Geschenkverbot weiterhin strikter und die Ausnahmen enger gehandhabt werden dürften. Sodann verbietet die Selbstregulierung im Rahmen der Unterstützung von Fort- und Weiterbildung die Mitfinanzierung jeglicher Rahmenprogramme (soziale Anlässe etc.), was das Gesetz noch zulässt, falls diese deutlich untergeordnet sind (vgl. Art. 6 Abs. 2 lit. d VITH). Spenden oder Veranstaltungssponsorings sind gemäss Selbstregulierung grundsätzlich nur möglich, wenn stets Selbstkostenbeiträge für die teilnehmenden Fachpersonen vorgesehen sind, was sich in dieser Klarheit nicht zwingend aus dem Gesetz ergibt. Zudem ist auf den Unterschied zwischen der in Art. 56 HMG festgeschriebenen Rabatttransparenz und der Transparenzinitiative gemäss PKK hinzuweisen. Während Erstere aufgrund wettbewerbsrechtlicher Überlegungen bewusst nicht im PKK geregelt wurde, ist Zweitere eine rein selbstregulative Vorgabe. Die Verpflichtung zur Veröffentlichung der Kooperationszuwendungen im Kontext verschreibungspflichtiger Arzneimittel geht also über Art. 56 HMG hinaus und ist von der Rabatttransparenz $\mathrm{zu}$ unterscheiden. Schliesslich ist die Selbstregulierung in vielerlei Hinsicht konkreter und erweist sich damit als griffige Hilfestellung in der Umsetzung der gesetzlichen Vorgaben. Zu erwähnen sind hier die inhaltlichen Vorgaben zur Ausgestaltung der Kooperationsvereinbarungen, die in Ziffer 41ff revidierter PK (revPK) viel detaillierter und konkreter aufgeführt werden als in Art. 7 VITH.

\section{Jüngste Revision}

Die Integritätsbestimmungen unter Ziffer 15ff revPK halten eingangs das Geschenkverbot als Grundsatz sowie die Ausnahmen von diesem fest (Ziffer 15.3 revPK). Die Ziffer wurde so umgestaltet, dass sie nun grundsätzlich dem Aufbau von Art. 55 HMG folgt. Ausgenommen vom Geschenkverbot sind Gegenstände, Informations- und Ausbildungsmaterialien von bescheidenem Wert (maximal 300 Franken pro Fachperson und Jahr), die ausschliesslich für die medizinische oder pharmazeutische Tätigkeit bestimmt sind und keinen Praxisbedarf abdecken. Schreibgeräte und -blöcke von bescheidenem Wert dürfen von Pharmaunternehmen nur im Rahmen von Veranstaltungen zur Verfügung gestellt werden, wenn sie dann weder mit Hinweisen auf das Pharmaunternehmen noch auf bestimmte Arzneimittel versehen sind. Sodann bleiben Unterstützungsbeiträge für Forschung, Weiter- und Fortbildung unter Einhaltung ausführlicher Vorgaben zulässig (vgl. dazu Ziffer 3 revPK). Angemessene Abgeltungen für gleichwertige Gegenleistungen - auch solche bei Bestellungen und Lieferungen von Arzneimitteln sowie beim Arzneimitteleinkauf gewährte Preisrabatte oder Rückvergütungen - bleiben ebenso statthaft, sofern sie keinen Einfluss auf die Wahl der Behandlung haben. Schliesslich bleibt die unentgeltliche Abgabe von Musterpackungen an Fachpersonen gemäss den Vorgaben von Swissmedic zulässig.

\section{Die Integritätsbestimmungen halten unter}

Ziffer 15ff revPK eingangs das Geschenkverbot als Grundsatz sowie die Ausnahmen von diesem fest.

Um klarzustellen, dass Zuwendungen für Mahlzeiten nur im Rahmen von Fachgesprächen und damit als stellvertretendes Honorar für die Erbringung einer Gegenleistung zulässig sind (vgl. Art. 7 VITH), wurden diese als eine eigenständige Integritätsvorschrift ausgestaltet. Gleichzeitig wurde der Höchstgrenzwert auf 100 Franken reduziert (vgl. Ziffer 15.4 revPK), womit auch hier die Selbstregulierung strikter als das Gesetz ist. Dieses erlaubt ein Überschreiten dieses Betrages, verlangt dann aber eine schriftliche Vereinbarung. Zudem kommt dieser Höchstsatz gemäss PK auch für Verpflegungen im Rahmen von Veranstaltungen zur Anwendung.

Die Unterstützung einer Kooperation mit einer Fachperson, Gesundheitsversorgungs- oder Patientenorganisationen soll durch mehrere Pharmafirmen erfol- 
gen, um den Einfluss eines einzelnen Unternehmens so gering wie möglich zu halten. Dieser im Umgang mit den Patientenorganisationen entwickelte Grundsatz wurde nun auf alle Kooperationspartner ausgeweitet und ein ausdrückliches Gebot des Multisponsoring formuliert (vgl. Ziffer 15.7 revPK/revPKK). Ausnahmen davon sind nur für Unterstützungen im Kontext von Seltenen Krankheiten denkbar.

Sodann soll sich Fachwerbung nur an diejenigen Fachpersonen oder Gesundheitsversorgungs-Organisationen richten, von denen vernünftigerweise angenommen werden kann, dass sie bestimmte Informationen zur Ausübung ihrer Tätigkeit benötigen oder daran interessiert sind. Alle Versandlisten müssen stets auf dem neuesten Stand gehalten werden. Wird die Streichung von einer verlangt, so muss ein Pharmaunternehmen umgehend dafür sorgen. Kommunikationen zu Werbezwecken mit Fax, E-Mail, automatisierten Anrufsystemen oder anderen digitalen Formen sollen - wenn immer möglich - mit vorheriger Genehmigung erfolgen (vgl. Ziffer 29 revPK). Auch bedarf die öffentliche Verwendung eines Logos sowie urheberrechtlich geschützten Materials einer Fachperson, Gesundheitsversorgungs-Organisation oder von Patientenorganisationen deren schriftlicher Genehmigung.

\section{Zuwendungen für Mahlzeiten sind nur im Rahmen von Fachgesprächen zulässig, der Höchstwert wurde auf 100 Franken reduziert.}

Pharmaunternehmen können weiterhin medizinische Weiter- und Fortbildung unterstützen, wenn sie die Förderung der wissenschaftlichen Kenntnisse und Kompetenzen von Fachpersonen bezweckt. Dabei können sie sich an verschiedenen Arten der Weiter- und Fortbildung beteiligen, solange diese keine Werbung dar- stellen. Allfällige werberische Aktivitäten müssen von der Weiter-/Fortbildungsveranstaltung klar getrennt sein (vgl. Ziffer 31 revPK). Von grosser Bedeutung in diesem Zusammenhang sind zudem die über die Selbstregulierung begründeten und mittlerweile in Art. 6 VITH verankerten Kostenbeteiligungsregeln der Fachpersonen (vgl. Ziffer 35 revPK).

\section{Würdigung}

Die Schweizer Pharma-Selbstregulierung ist eine Erfolgsgeschichte, die auch nach der HMG-Revision fortgeschrieben wird. Die Kooperation zwischen der Industrie und verschiedenen Akteuren im Gesundheitswesen ist für die Entwicklung neuer Therapien

\section{Eine auf hohen ethischen Standards beruhende}

Kooperation ist in letzter Konsequenz im Interesse der Patientinnen und Patienten.

von grosser Bedeutung. Dies zeigt aktuell auch die Herausforderung der Corona-Pandemie: Ohne einen vernetzten Informationsaustausch und Kooperationen zwischen Forschung, Industrie und Gesundheitsfachleuten würden die Erkenntnisse weniger rasch erarbeitet und die Entwicklung von Behandlungsansätzen viel später klinisch getestet werden können. Eine auf hohen ethischen Standards beruhende Kooperation ist deshalb in letzter Konsequenz im Interesse der Patientinnen und Patienten. Gerade in der raschen Umsetzung dieser Vorgaben erweist sich die Pharma-Selbstregulierung als effizient und wirkungsvoll.

Bildnachweis

(c) Pressmaster | Dreamstime.com (Symbolbild)
Jürg Granwehr

lic. iur. Rechtsanwalt

Bereichsleiter Pharma

\& Recht

Tel. 0443681728

juerg granwehr[at]

scienceindustries.ch

scienceindustries -

Wirtschaftsverband

Chemie Pharma Life

Sciences

Nordstrasse 15

Postfach

CH-8021 Zürich

\section{Das Wichtigste in Kürze}

- Am 1. Januar 2021 werden die revidierten Kodizes der Pharma-Selbstregulierung in Kraft treten.

- Das selbstregulative Geschenkverbot dürfte strikter gehandhabt werden als Art. 3 VITH, insofern als die Ausnahmen enger gefasst bleiben dürften.

- Zuwendungen für Mahlzeiten sind nur im Rahmen von Fachgesprächen oder von Veranstaltungen zulässig und dürfen maximal 100 Franken kosten.

- Ein allgemeines Gebot des Multisponsorings wurde explizit festgeschrieben.

- Die Kostenbeteiligungsregeln gelten umfassend sowohl bei der direkten Unterstützung von Fachpersonen als auch bei der indirekten Unterstützung über eine Organisation.

- Es dürfen keinerlei Rahmenprogramme bei Veranstaltungen vorgesehen resp. unterstützt werden.

\section{L'essentiel en bref}

- Le Code pharmaceutique révisé destiné à l'autorégulation du secteur entrera en vigueur le $1^{\text {er }}$ janvier 2021.

- Dans celui-ci, l'interdiction de cadeaux devrait être plus stricte que dans l'art. 3 OITPTh, dans la mesure où les exceptions devraient être plus restrictives.

- Les indemnités de repas ne sont autorisées que dans le cadre de discussions de professionnels et de manifestations et sont plafonnées à 100 francs.

- Le principe général du multisponsoring est explicitement inscrit dans le Code.

- Les règles de participation aux coûts s'appliquent à la fois au soutien direct aux professionnels et au soutien indirect par l'intermédiaire d'une organisation.

- Aucun programme-cadre ne peut être prévu ni soutenu lors de manifestations. 